\title{
In vivo versus in vitro protein abundance analysis of Shigella dysenteriae type 1 reveals changes in the expression of proteins involved in virulence, stress and energy metabolism
}

Srilatha Kuntumalla ${ }^{1 \dagger}$, Quanshun Zhang ${ }^{2 \dagger}$, John C Braisted ${ }^{1}$, Robert D Fleischmann ${ }^{1}$, Scott N Peterson', Arthur Donohue-Rolfe ${ }^{2}$, Saul Tzipori ${ }^{2}$ and Rembert Pieper ${ }^{1 *}$

\begin{abstract}
Background: Shigella dysenteriae serotype 1 (SD1) causes the most severe form of epidemic bacillary dysentery. Quantitative proteome profiling of Shigella dysenteriae serotype 1 (SD1) in vitro (derived from LB cell cultures) and in vivo (derived from gnotobiotic piglets) was performed by 2D-LC-MS/MS and APEX, a label-free computationally modified spectral counting methodology.

Results: Overall, 1761 proteins were quantitated at a 5\% FDR (false discovery rate), including 1480 and 1505 from in vitro and in vivo samples, respectively. Identification of 350 cytoplasmic membrane and outer membrane (OM) proteins (38\% of in silico predicted SD1 membrane proteome) contributed to the most extensive survey of the Shigella membrane proteome reported so far. Differential protein abundance analysis using statistical tests revealed that SD1 cells switched to an anaerobic energy metabolism under in vivo conditions, resulting in an increase in fermentative, propanoate, butanoate and nitrate metabolism. Abundance increases of transcription activators FNR and Nar supported the notion of a switch from aerobic to anaerobic respiration in the host gut environment. High in vivo abundances of proteins involved in acid resistance (GadB, AdiA) and mixed acid fermentation (PfIA/PflB) indicated bacterial survival responses to acid stress, while increased abundance of oxidative stress proteins (YfiD/ YfiF/SodB) implied that defense mechanisms against oxygen radicals were mobilized. Proteins involved in peptidoglycan turnover (MurB) were increased, while $\beta$-barrel OM proteins (OmpA), OM lipoproteins (NIpD), chaperones involved in OM protein folding pathways ( $\mathrm{YraP}, \mathrm{NlpB}$ ) and lipopolysaccharide biosynthesis (Imp) were decreased, suggesting unexpected modulations of the outer membrane/peptidoglycan layers in vivo. Several virulence proteins of the Mxi-Spa type III secretion system and invasion plasmid antigens (Ipa proteins) required for invasion of colonic epithelial cells, and release of bacteria into the host cell cytosol were increased in vivo.

Conclusions: Global proteomic profiling of SD1 comparing in vivo vs. in vitro proteomes revealed differential expression of proteins geared towards survival of the pathogen in the host gut environment, including increased abundance of proteins involved in anaerobic energy respiration, acid resistance and virulence. The immunogenic OspC2, OspC3 and IpgA virulence proteins were detected solely under in vivo conditions, lending credence to their candidacy as potential vaccine targets.
\end{abstract}

\footnotetext{
* Correspondence: rpieper@jcvi.org

† Contributed equally

'Pathogen Functional Genomics Resource Center, J. Craig Venter Institute,

9704 Medical Center Drive, Rockville, MD 20850, USA

Full list of author information is available at the end of the article
} 


\section{Background}

The Gram-negative bacterium Shigella dysenteriae serotype 1 (SD1) is among the most virulent serotypes of the four Shigella (S.) species (S. dysenteriae, S. flexneri, S. son$n e i$ and $S$. boydii). SD1 is a causative agent of shigellosis, a severe form of epidemic bacillary dysentery in humans and primates $[1,2]$. Shigellosis is most prevalent in underdeveloped countries, with a mortality rate of $10-15 \%$ when untreated, killing about 1.1 million people of the roughly 120 million cases each year http://www.who.int/ vaccine_research/diseases/diarrhoeal/en/index6.html. SD1 has an extremely low infectious dose of 10-100 organisms which has contributed to causing pandemic Shiga dysentery in several continents including Asia, Africa and Central America [2]. In addition to having a low infectious dose, multi-drug antibiotic resistance to more than six types of antibiotics (tetracycline, streptomycin, chloramphenicol, etc.) has developed in several Shigella serotypes [3]. S. dysenteriae is also very closely related to Escherichia (E.) coli, with certain strains of E. coli (Shiga toxin-producing E. coli, or STEC) producing the potent Shiga toxins (Stx) of which Stx1 is produced by SD1 as well [4]. Shiga toxin causes cell death primarily in the microvascular endothelium. A vaccine that is protective against Shigella serotypes is of utmost importance, and several attenuated vaccines are currently being developed and tested in human volunteers.

Components of the Type Three Secretion System (TTSS) encoded by a virulence plasmid are also involved in the pathogenesis of shigellosis [5]. Also called the Mxi-Spa system in Shigella, the TTSS is responsible for triggering entry into host epithelial cells and apoptosis in macrophages $[6,7]$. The TTSS is activated upon contact with host cells, leading to the integration of translocators in the host cell membranes which then promotes transit of effectors into host cells [8]. The TTSS and effector proteins thereby play an important role in infection and intra- and inter-cellular spreading of bacterial cells in the host intestinal epithelium [9]. O-antigens present in the cell surface lipopolysaccharide (LPS) of Shigella also contribute to its virulence [2]. The Shigella O-antigen comprises of a toxic lipid A moiety embedded in the bacterial outer membrane, a core sugar region and an exposed terminal O-polysaccharide. In SD1, the O-polysaccharide consists of tetrasaccharide repeats that contain repeat units of three rhamnose residues and one $\mathrm{N}$-acetylglucosamine [2]. Enzymes essential for Oantigen biosynthesis are mostly encoded by chromosomal genes such as galETKM and $r f b B D A C X$ [10]. The terminal $O$-polysaccharide structures vary greatly among Shigella, thereby giving rise to the immunological specificity that has resulted in distinct serotypes. Although attenuated Shigella strains expressing genetically engineered $\mathrm{O}$-antigens have been tested as vaccine candidates, an effective vaccine against Shigella remains elusive [2], possibly due to the diversity of the $\mathrm{O}$-antigens.

Comprehensive proteomic profiling has the potential to identify novel virulence factors in Shigella that could form potential vaccine or therapeutic targets. Proteomic surveys of Shigella have mainly focused on S. flexneri, which causes endemic shigellosis. Extensive 2D-LC-MS/MSbased profiling of the S. flexneri membrane proteome by Wei et al. resulted in the identification of more than 600 S. flexneri proteins including ca. 200 integral and outer membrane proteins [11]. Immunoproteome analyses of $S$. flexneri identified several membrane proteins as being antigenic including OmpA, IpaD, Spa33, TolC and YaeT $[12,13]$. The $S$. dysenteriae strain Sd197 was the first S. dysenteriae genome to be sequenced [14], and included sequences of the chromosome, a large virulence-associated plasmid (pSD1_197) and a small plasmid (pSD197_Spa). This annotated SD1 genome was exploited in a comprehensive proteomic survey of $S$. dysenteriae strain Sd1617 via 2D gel electrophoresis which resulted in the identification of 1061 distinct gene products [15]. Immunoproteome analysis of SD1 Sd1617 identified seven proteins including type III secretion system effectors OspC2 and IpaB as antigens. In this report, a quantitative global proteomic analysis of SD1 cells grown to stationary phase in culture (in vitro) vs. SD1 cells isolated from mammalian host environment (in vivo) was performed using 2D-LCMS/MS and APEX, a label-free computationally modified spectral counting method [16]. Data from the SD1 in vitro and in vivo proteomes was analyzed for differential protein expression in the context of virulence and survival in the host.

\section{Methods}

\section{Materials and reagents}

All reagents for protein extraction from cell lysates and protein analysis by mass spectrometry (MS) were used as previously described $[15,17]$. RNase, DNase I (bovine pancreas), triethyl ammonium bicarbonate (TAB) buffer used for tryptic digestion, TCEP (Tris(2-carboxyethyl)phosphine) used as a reducing agent and the bicinchoninic acid (BCA) protein assay kit to estimate protein concentrations were purchased from Sigma-Aldrich (St. Louis, MO). The alkylating agent MMTS (methyl methanethiosulfonate) was purchased from Pierce (Rockford, IL). Sequencing grade porcine trypsin was obtained from Promega (Madison, WI). Triton X-100 was purchased from Calbiochem (LaJolla, CA). SDS-PAGE was performed according to instructions from Invitrogen.

\section{Bacterial strains and culture conditions}

The strain Sd1617 of Shigella dysenteriae serotype 1 (SD1) strain was grown on tryptic soy agar plates (TSA) 
containing $0.05 \%$ Congo Red (w/v). SD1 in vitro samples were prepared by inoculating a single colony into LuriaBertani (LB) medium grown to stationary phase at $37^{\circ} \mathrm{C}$ with agitation. The bacteria were harvested by centrifugation and washed twice with ice-cold PBS $(6,000 \times g$, $15 \mathrm{~min})$ at $4^{\circ} \mathrm{C}$.

The inoculum for in vivo experiments was prepared by growing a typical SD1 colony selected from a TSA plate in LB medium overnight. Gnotobiotic piglets used for the animal experiments were delivered by Caesarian section at Tufts University Cummings School of Veterinary Medicine. Of several animals inoculated with SD1, three piglets were chosen for isolation of SD1 bacterial cells from the intestine in this comparative study. One of the piglets inoculated with $1 \times 10^{8} \mathrm{SD} 1$ cells developed diarrhea $24 \mathrm{~h}$ later and was euthanized $4 \mathrm{~d}$ later when the gut contents were collected for bacterial purification. Another piglet inoculated with $5 \times 10^{8}$ SD1 cells developed diarrhea within $18 \mathrm{~h}$ and was euthanized $3 \mathrm{~d}$ postinoculation. A third piglet inoculated with $5 \times 10^{9} \mathrm{SD} 1$ cells developed diarrhea within $20 \mathrm{~h}$ and the gut contents collected $2 \mathrm{~d}$ post-inoculation. SD1 bacterial cells were isolated from the gut contents as described previously [15]. Briefly, the gut contents from cecum and colon were pooled and transferred to sterile histological cups placed on ice, suspended in ice-cold PBS at $4{ }^{\circ} \mathrm{C}$ and pelleted at $5,000 \times g$. After resuspension of the pellet in $65 \%$ isotonic Percoll solution and centrifugation at $14,500 \times g$, the bacterial layer near the bottom was collected using a 3-5 $\mathrm{ml}$ syringe with needle. The bacteria were washed twice with ice-cold PBS at $4^{\circ} \mathrm{C}$ and processed for proteomic analysis.

\section{Lysis of S. dysenteriae cells and trypsin digestion of extracted proteins}

After the PBS wash steps, bacterial cell pellets from in vitro or in vivo culture conditions were re-suspended in a hypotonic lysis buffer composed of $25 \mathrm{mM}$ Tris- $\mathrm{HCl}$ ( $\mathrm{pH}$ 7.8) with $150 \mu \mathrm{g} / \mathrm{mL}$ lysozyme, $0.05 \%$ Triton X-100, $5 \mathrm{mM}$ EDTA, protease inhibitors ( $1 \mathrm{mM}$ benzamidine and AEBSF) for $30 \mathrm{~min}$ at room temperature (RT) with gentle agitation. The samples were then placed at $-80^{\circ} \mathrm{C}$ until further processing. For nucleic acid digestion, bacterial samples suspended in the lysis buffer were thawed and gently agitated for $1 \mathrm{~h}$ at RT after the addition of DNase I, RNase and leupeptin $(10 \mu \mathrm{g} / \mathrm{mL}$ each $)$ and $20 \mathrm{mM} \mathrm{MgCl}_{2}$. Cell lysates were centrifuged at 16,000 × $g$ for $30 \mathrm{~min}$ at $4^{\circ} \mathrm{C}$, and the supernatants containing bacterial cell lysate proteins were recovered.

Following cell lysis, the extracted bacterial proteins were precipitated in six volumes of ice-cold acetone at $-20^{\circ} \mathrm{C}$ for at least $1 \mathrm{~h}$. Acetone-precipitated proteins were recovered as a pellet after centrifugation at $5,000 \times g$ for $10 \mathrm{~min}$. The protein pellet was resuspended in $0.1 \mathrm{M}$
TAB buffer, $\mathrm{pH} 8.5$, and the total protein concentration measured using the BCA assay. Proteins were denatured in $0.1 \%$ SDS and reduced using $5 \mathrm{mM}$ TCEP for $1 \mathrm{~h}$ at $37^{\circ} \mathrm{C}$, followed by alkylation using $10 \mathrm{mM}$ MMTS for $1 \mathrm{~h}$ at RT [18]. In-solution trypsin digestion of the complex protein mixture was performed by the addition of trypsin at $1: 25$ for $5 \mathrm{~h}$ at $37^{\circ} \mathrm{C}$ followed by $1: 50$ digestion overnight. The tryptic digested samples were applied to SDSPAGE to check for extensive digestion.

\section{Mass spectrometry analysis of tryptic peptides}

Methods for mass spectrometry (MS) analysis were previously described in detail [17]. Briefly, tryptic peptide digests $(c a .100 \mu \mathrm{g})$ were fractionated by 2D-LC-MS/MS, first using a Polysulfoethyl-A SCX column $(4.6 \times 50 \mathrm{~mm}$, Nest Group, USA) followed by an Agilent 1100 series solvent delivery system (Agilent, Palo Alto, CA) online with a nano-electrospray LC-MS/MS system (LTQ-IT mass spectrometer, Thermo-Finnigan, San Jose, CA). SCX fractions were delivered from 96-well plates onto a PicoTip microcapillary reversed-phase column (BioBasic $\mathrm{C}_{18}$, $75 \mu \mathrm{m} \times 10 \mathrm{~cm}$, New Objective, Woburn, MA) at a flow rate of $350 \mathrm{~nL} / \mathrm{min}$. Spectra were acquired in automated MS/MS mode with the top five parent ions selected for fragmentation using collision energy of 35\%. LC-MS/MS was performed in three sequential $\mathrm{m} / \mathrm{z}$ subscans (300$650,650-900,900-1500 \mathrm{~m} / z)$ to increase the sampling depth [16].

MS and MS/MS data from sequential runs were combined for search against the latest release of the $S$. dysenteriae Sd197 genome database in NCBInr using the Mascot search engine v.2.2 (Matrix Science, London, UK). This database contained 4502 protein sequences, including 231 proteins encoded by the two SD1 plasmids. Mascot search parameters allowed for tryptic specificity of up to one missed cleavage, with methylthio-modifications of cysteine as a fixed modification and oxidation of methionine as a variable modification. The LTQ search parameters for $+1,+2$ and +3 ions included mass error tolerances of $\pm 1.4 \mathrm{Da}$ for peptide ions and $\pm 0.5 \mathrm{Da}$ for fragment ions. The false discovery rate (FDR) for peptide identifications was determined using the Mascot decoy database search option, with searches against a randomized S. dysenteriae Sd197 protein decoy database. Mascot search results of replicate 2D-LC-MS/MS experiments were further validated by estimating the FDR [19] via PeptideProphet ${ }^{\mathrm{TM}}$ and ProteinProphet ${ }^{\mathrm{TM}}$ [20] which are part of the Trans-Proteomic Pipeline (TPP) available at http://tools.proteomecenter.org/wiki/ index.php?title=Software:TPP.

APEX quantitation of SD1 cell lysate LC-MS/MS datasets APEX quantitation of SD1 proteins was performed using the APEX Quantitative Proteomics Tool [21]v.1.1 as 
described previously [17]. Briefly, three steps were performed, building a SD1 training dataset, computing SD1 protein $O_{i}$ (expected number of unique proteotypic peptides for protein $i$ ) values, and calculating SD1 protein APEX abundances. Proteins in the training dataset were comprised of the 100 most abundant SD1 proteins based on high spectral counts per protein and high protein and peptide identification probabilities [22]. The training dataset. ARFF file was constructed based on 35 peptide physicochemical properties deemed significant for the computational prediction of proteotypic peptides $[16,23]$. To compute SD1 protein $O_{i}$ values, the Random Forest classifier algorithm was applied to the SD1 training dataset constructed in the previous step, and then to all tryptic peptides generated in silico from the SD1 proteome to enable computation of SD1 protein $O_{i}$ values. APEX abundances of the SD1 proteins observed by 2DLC-MS/MS were calculated using the protXML files generated from the PeptideProphet ${ }^{\mathrm{TM}}$ and ProteinProphet $^{\mathrm{TM}}$ validation of the Mascot search results and the SD1 protein $O_{i}$ values. While data from the technical replicates (three to five) for each of the three biological samples were pooled in the analysis, data from the biological replicates were analyzed separately under in vitro and in vivo conditions. A $<5 \%$ FDR was chosen, along with a normalization factor of $2.5 \times 10^{6}$. The normalization factor in the APEX tool is equivalent to the term $C$ in the APEX equation [16], which represents the total concentration of protein molecules per cell. Since $S$. $d y s-$ enteriae is closely related to $E$. coli, the total number of protein molecules/cell estimated at $2-3 \times 10^{6}$ for $E$. coli [16] was used as a normalization factor in the APEX abundance measurements of $S$. dysenteriae proteins.

\section{Bioinformatic analysis tools}

In silico predictions of subcellular protein localizations were obtained using PSORTb v.2.0 searches [24] of the S. dysenteriae Sd197 proteins. In cases where the PSORTb analysis was inconclusive, the datasets were queried by five other algorithms (SignalP [25], TatP [26], TMHMM [27], BOMP [28] and LipoP [29]) to predict motifs for export signal sequences, TMD proteins and lipoproteins in SD1 proteins.

\section{Statistical analysis, clustering and pathway analysis of SD1 proteomic datasets}

Differential protein expression analysis of the in vitro vs. in vivo proteomes was examined using a two-tailed Z-test [16] incorporated into the APEX tool [21]. The p-values from the Z-test obtained for the proteins common to the in vitro and in vivo samples were subjected to the Benjamini-Hochberg (B-H) multiple test correction available from the open source $\mathrm{R}$ statistical package http://www.r-project.org to estimate the false discovery rate (FDR). Further statistical analysis and clustering of the data were performed using the MeV v.4.4 (Multiexperiment Viewer) software tool, an application designed for detailed statistical analysis of large-scale quantitative datasets [30,31]. A two-class SAM (Significance Analysis for Microarrays) was performed, and a heat map generated by clustering the data using HCL (Hierarchial Clustering) and Euclidean distance in $\mathrm{MeV}$. To determine the reproducibility of the datasets, a pairwise Pearson's correlation plot was constructed to correlate protein abundance values obtained for each protein from replicate analyses. For pathway analysis, the $S$. dysenteriae Sd197 KEGG pathway was obtained from the KEGG database [32]. The KEGG pathway was loaded into Katsura v.1.0 (JCVI), which is an open source software application for exploring the KEGG metabolic pathway coverage and expression available at http://pfgrc.jcvi.org/ index.php/bioinformatics/katsura.html. To identify the SD1 metabolic pathways and functional proteins that were altered under in vivo conditions as compared to in vitro conditions, each pathway was examined for proteins exhibiting higher or lower protein abundance values based on the two-tailed Z-test analysis.

\section{Results and Discussion}

\section{Global profiling of $S$. dysenteriae strain Sd1617 in vitro} and in vivo proteomes

Shigella dysenteriae serotype 1 (SD1), which possesses the cytotoxic Shiga toxin (Stx), causes deadly epidemics in many poor countries [14]. However, no effective vaccine for this pathogenic organism is currently available although there are several attenuated strains at different stages of development [2]. Proteomic analysis of S. dysenteriae is a strategy to identify novel vaccine and therapeutic drug targets. A gnotobiotic piglet model was recently developed [33] to serve as an alternative to a primate model to study infections with the highly hostspecific pathogen $S$. dysenteriae [15,34]. SD1 bacterial cells were collected from stationary phase suspension cultures in LB broth (referred to as 'in vitro') and from the gut of several infected gnotobiotic piglets (referred to as 'in vivo'). The lack of microflora in gnotobiotic animals and the ability to recover more than $10^{9}$ purified SD1 bacteria from in vivo conditions allowed unique studies of the nature of the pathogen's direct interaction with the host tissue in the absence of other interfering microflora.

A preliminary 2D gel-based survey of the SD1 proteome from the piglet intestinal environment was reported previously [15]. Here, the scope of the differential proteomic analysis was expanded using three to five technical and three biological replicates from both in vitro and in vivo groups. We resorted to a strategy combining the benefits of 2D-LC-MS/MS for a comprehensive coverage of 
proteins, and APEX (a modified spectral counting method for protein expression measurements derived from LCMS/MS datasets). The in vitro analysis resulted in the identification of 1480 proteins while the in vivo analysis identified 1505 proteins at a $5 \%$ false discovery rate (FDR). 1224 proteins were common to both samples, with 256 and 281 proteins unique to the in vitro and in vivo analyses, respectively (Figure 1). Genome sequencing of the strain Sd197 suggested 4271 chromosomal ORFs, 223 plasmid pSD1_197-encoded ORFs and 8 plasmid pSD197_spA-encoded ORFs [14]. Combining LC-MS/MS data from all experiments and assuming a 5\% FDR, 1761 proteins comprising $39 \%$ of the SD1 proteome were identified across a wide $\mathrm{M}_{\mathrm{r}}(4.3$ - $176.5 \mathrm{kDa})$ and $\mathrm{pI}(3.59$ 11.84) range (Additional File 1, Table S1). 105 and 95 proteins were identified based on a single peptide match from the in vitro and in vivo analyses, respectively. Since small or low abundance proteins are frequently identified by one or two peptides [19], validation of the single peptide match proteins was performed by validating the spectrum manually. Of the 231 proteins encoded by the two plasmids pSD1_197 and pSD197_spA, 66 and 3 proteins were identified, respectively. This included $15 \mathrm{Mxi}-\mathrm{Spa}$ proteins and 16 effectors/chaperones of the type III secretion system (TTSS) clustered in the ipa gene locus of pSD1_197. Wei et al. [11] identified 45 of the orthologous $S$. flexneri proteins expressed from the plasmid pCP301, including $8 \mathrm{Mxi}$-Spa proteins and 11 effectors/chaperones.

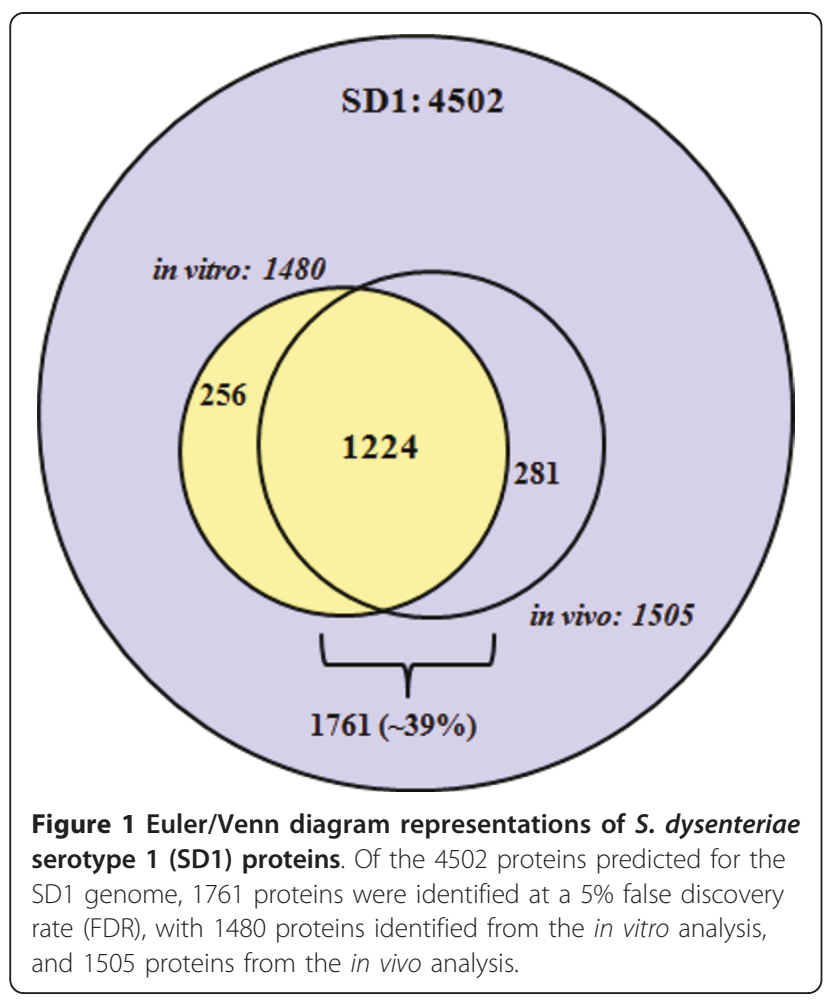

The comparison supports the notion that expression of these genes is important in the proper functioning of the TTSS of both Shigella species.

Subcellular localizations (SCL) of all 1761 identified SD1 proteins were determined, either based on in silico predictions by the tool PSORTb or by the combination of short motifs recognized in protein sequences by six different algorithms (SignalP, TatP, TMHMM, BOMP, LipoP and KEGG pathway role). Data from the latter categorization are displayed in Figure 2, with most proteins (1310) being assigned to the cytoplasm. As membrane proteins are often of particular interest in the context of virulence, they were also selectively surveyed in a study on S. flexneri $2 \mathrm{a}$ [11], yielding approximately 35 outer membrane (OM) and 159 integral cytoplasmic membrane (CM) proteins. SCL prediction of our data yielded 350 membrane proteins (including $108 \mathrm{OM}$ and $242 \mathrm{CM}$ proteins), contributing to an extensive survey of the Shigella membrane proteome. Many peripheral, integral and lipid-anchored membrane proteins could also be quantitated applying the APEX tool. This is a marked advantage of 2D-LC-MS/MS over 2D gel-based proteomic surveys. For example, we were able to obtain quantitative estimates for numerous membrane proteins, some of them part of complexes. This included 7 of the 8 FOF1 ATP synthase subunits predicted for SD1 http://biocyc.org, 11 of the $13 \mathrm{NADH}$ dehydrogenase (Nuo) subunits, all three formate dehydrogenase subunits (FdoG/H/I), all four cytochrome oxidase subunits (CydA/B/C/D), $\beta$-barrel OM porins (OmpA, OmpC, OmpX), multidrug efflux transporters (MdlA, MdlB,

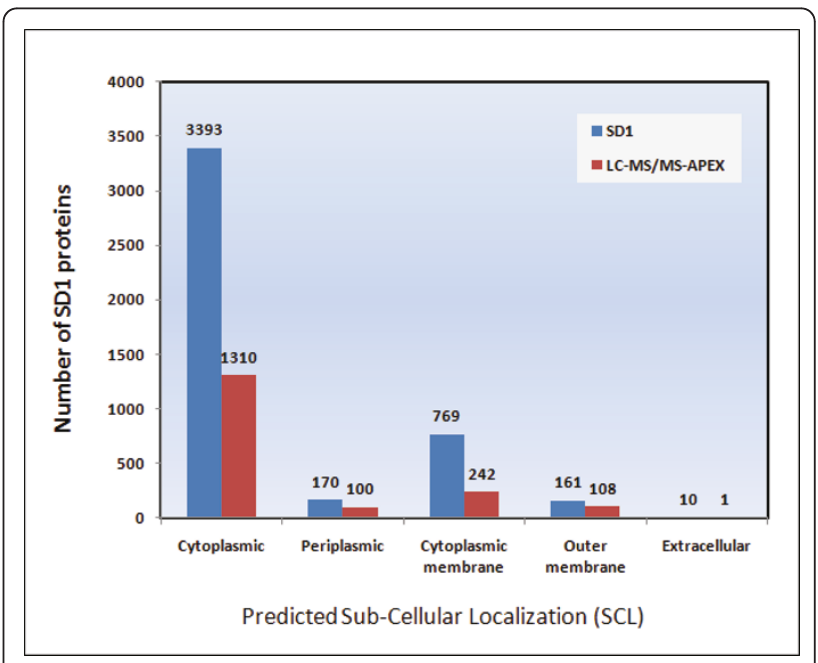

Figure 2 Sub-cellular localization (SCL) of SD1 proteins. SCL of 4502 proteins encoded by the SD1 genome was predicted using the bioinformatic algorithms PSORTb, SignalP, TatP, TMHMM, BOMP, LipoP and KEGG. 350 outer and inner membrane proteins

corresponding to $\mathrm{ca}$. $38 \%$ of the SD1 membrane proteome, and 1410 cytoplasmic and periplasmic proteins representing ca. 39\% of SD1 soluble proteins were identified. 
YdhE, YhiU, EmrA, EmrY) and 15 structural components of the bacterial Mxi_Spa apparatus. Most proteins or their orthologs which were described as being immunogenic by Ying et al. [12,35] in S. flexneri and Pieper et al. in $S$. dysenteriae (15), were also identified in this SD1 dataset (OmpA, YaeT, OppA, DnaK, ClpB, Pgm, AtpA, AtpD, LpdA, Gnd, Tst, MglB, FusA, ManX, TolC, UshA, OspC2, VirB and IpaB).

Highly abundant SD1 proteins, in vivo and in vitro, were implicated in energy/carbon metabolism and protein synthesis. This included glycolytic enzymes (PckA, GapA, Tpi, Fba, Pgk, GpmA, Eno), elongation factors (FusA, TufA, Tsf), several ribosomal protein subunits (RpsD/K/ $\mathrm{M}, \mathrm{RplC} / \mathrm{D} / \mathrm{E}, \mathrm{RpmC} / \mathrm{D} / \mathrm{J})$, and stress response proteins (WrbA, AhpC, SodB). Proteins with global regulatory functions in the cellular stress response were identified in vivo as well as in vitro (Hns, RpoS and CpxR). In summary, SD1 cells produced proteins essential for growth and cell integrity (energy generation, protein synthesis, cell envelope structure) as well as response to cellular and environmental stresses in high abundance.

\section{Differential abundance analyses of the SD1 in vitro and in vivo proteomes}

Data from three biological replicates pertaining to in vivo and in vitro conditions were subjected to statistical analyses. The biological replicate analyses were pooled for the Z-test, and analyzed separately by the SAM test. Differential expression analysis of the in vitro vs. in vivo proteomes using a two-tailed Z-test resulted in $c a$. 300 proteins identified as being differentially abundant at a $99 \%$ confidence level (Figure 3), while the SAM test identified ca. 90 differentially expressed proteins (Additional File 2, Table S2). As the SAM test takes into account the biological variability between replicates, it is more conservative at estimating the differential protein expression given the dynamic range of the biological data which may inflate variance measures. The Benjamini-Hochberg (B-H) multiple test correction performed on the 1224 proteins common to the in vitro and in vivo samples estimated the FDR at $<5 \%$ for the $c a$. 300 differentially expressed proteins identified from the Z-test (Additional Files 1 and 2, Tables S1 and S2). Hierarchial clustering of the data resulted in several major clusters of similarly expressed proteins (Figure 4). Selection of two clusters magnified in Figure 4 was based on biological interest in the set of proteins that exhibited differential abundance values. For example, one of the clusters harbored numerous ribosomal proteins and several Ipa/Ipg host cell invasion proteins, all of which were clearly increased in abundance in vivo. Another cluster harbored several enzymes indicative of the shift from aerobic to anaerobic energy generation. Protein functional role categories of the differentially expressed proteins were assigned according to the CMR database http://cmr.jcvi.

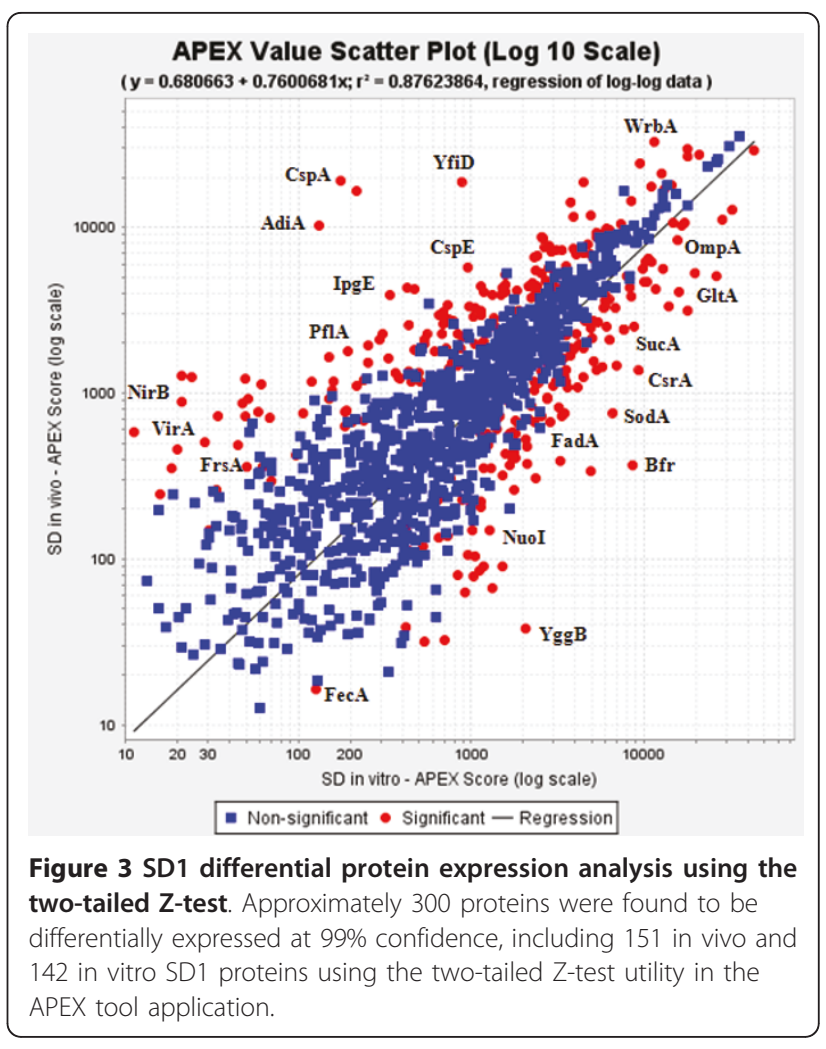

org and are displayed in Figure 5. Several cellular processes appeared to be activated in the intestinal environment comparing the percentage of abundance-changed proteins per role category in both the in vitro (Figure $5 \mathrm{a}$ ) and the in vivo (Figure 5b) groups. Most striking were the changes in protein synthesis $(0.6 \%$ vs. $18.1 \%$ in vitro and in vivo, respectively) and purine, pyrimidine and nucleotide biosynthesis (1.2\% vs. 5.8\%). In contrast, activity decreases in vivo were denoted for regulatory processes (4.9\% vs. $1.8 \%)$, cell envelope functions (5.6\% vs. $2.3 \%$ ) and transport (10.5\% vs. $7 \%)$. Overall, the graphic in Figure 5 clearly illustrates that the SD1 cells adapt to the host intestinal environment by alternating a multitude of their cellular pathways and processes.

Differential expression analysis of the APEX datasets revealed several biochemical processes that appeared to be important for the pathogen to infect the piglets and to survive in their intestinal environment. Strongly altered abundances in the in vivo environment pertained to proteins involved in mechanisms of acid resistance (GadB, AdiA, HdeB, WrbA), the switch from aerobic to anaerobic respiration and mixed acid fermentation (PflA, PflB, PykF, Pta), oxidative stress (YfiD, YfiF, SodB) and other general cellular stress responses involving cold and heat shock proteins (CspA, CspE, ClpB). The in vivo responses suggested enhanced bacterial stress under oxygen- and nutrient-limited conditions in 


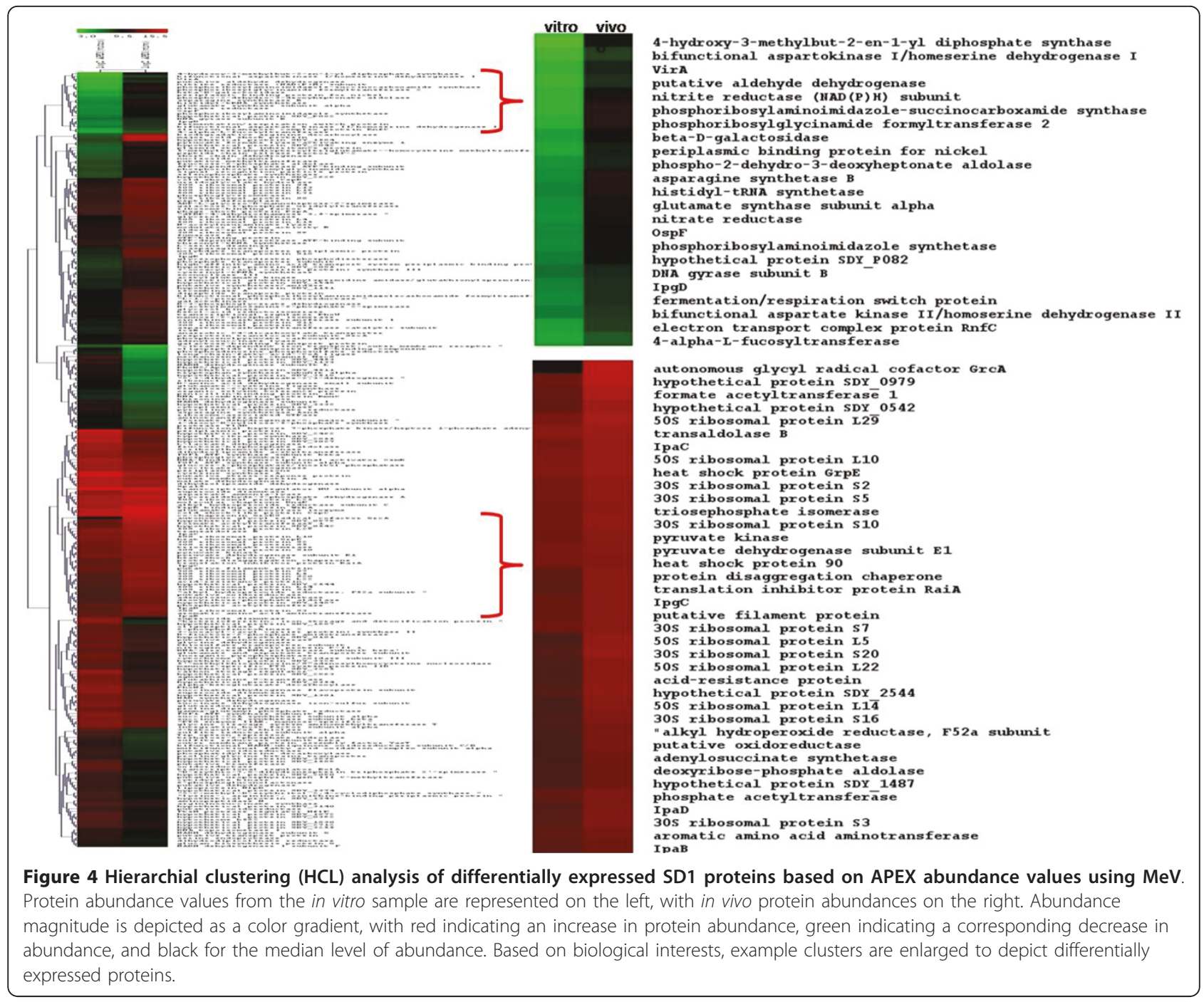

the host gut environment. In contrast, the in vitro proteome was defined by high abundances of enzymes involved in fatty acid oxidation (FadA, FadB, FadD, etc.) and aerobic respiration (GltA, IcdA, SdhA, SucA, etc.). Several proteins linked to the type III secretion apparatus were increased in abundance, or found solely under in vivo conditions including chaperones for effector proteins (IpgA/B/C/D/E), transcription activators (VirB), translocators (IpaA/B/C/D) and effectors (IcsB, VirA, OspF).

\section{Transition from aerobic to anaerobic respiration and fermentation in SD1 bacterial cells in vivo} The pathogenic $S$. dysenteriae is a facultative anaerobe which can switch to an anaerobic energy metabolism when starved of oxygen in the host large intestine. Proteins involved in energy metabolism formed the largest category of abundance-changed proteins under both in vitro and in vivo conditions (Figure 5), indicative of the impact of the intestinal environment on the SD1 cell's energy generation pathways. We have previously reported in a $2 \mathrm{D}$ gel-based proteomic analysis that the intestinal environment resulted in a shift from aerobic respiration to fermentation in SD1 cells (15). A more comprehensive dataset was obtained in this study and highlights the advantages of 2D-LC-MS/MS and APEX over differential 2D gel display. The former approach is not only more sensitive, as it strongly increased the number of profiled low abundance proteins, but also revealed marked advantages via the quantitation of hydrophobic CM and OM proteins.

It was confirmed that most of the tricarboxylic acid (TCA) cycle enzymes were strongly decreased in SD1 cells in vivo, such as GltA, IcdA, SucA, SucB, SucC, SucD, SdhA, SdhB and Mdh. The abundance changes of these and following enzymes are provided in Additional File 1, Table S1. The TCA cycle was clearly less active under anaerobic (in vivo) than aerobic/microaerophilic 


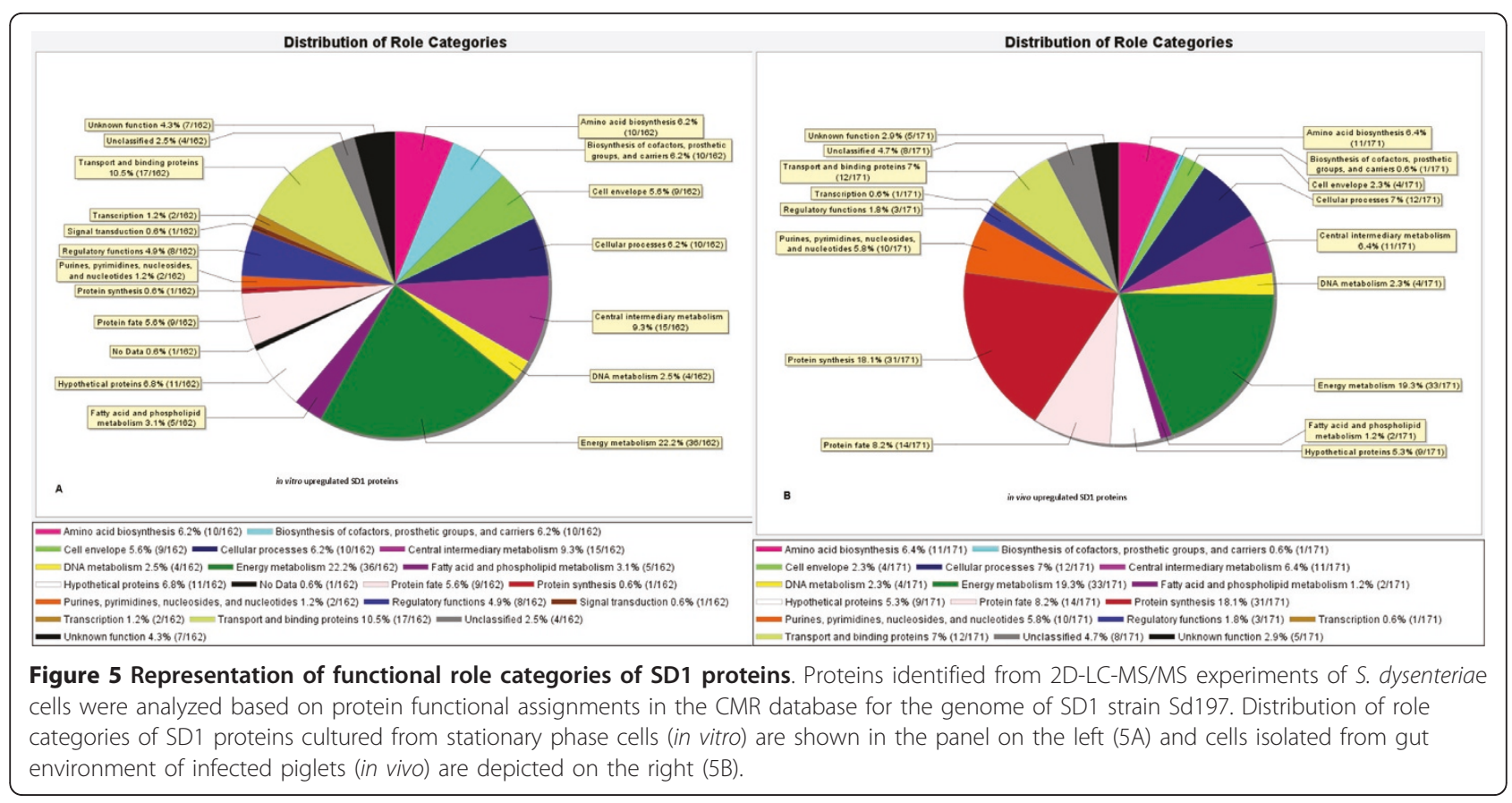

(in vitro) conditions. New evidence was obtained that the major enzyme complex contributing electron donors in the aerobic respiratory chain, NADH:ubiquinone dehydrogenase I, was markedly less active in vivo. Nearly all of the subunits (NuoA/B/C/E/F/G/H/I/K/L/ $\mathrm{N})$ were decreased in abundance in vivo. Likewise, a second major electron donor enzyme complex known to be active under aerobic conditions in $E$. coli, succinate dehydrogenase, featured strong decreases in vivo (SdhA/ $\mathrm{B} / \mathrm{C} / \mathrm{D})$. The cytochrome b562 protein CybC was also strongly decreased in vivo.

The question arose as to which electron donor complexes substituted for Sdh and Nuo in vivo to support anaerobic and microaerobic respiration. Surprisingly, subunits of formate dehydrogenase $(\mathrm{FdoG} / \mathrm{H} / \mathrm{I})$ were moderately decreased in abundance in vivo, whereas Fdn was not detected at all. Fdn is purportedly a selective electron donor for anaerobic respitration http://ecocyc.org. FNR (fumarate and nitrate reductase regulator) and NarP, both components of the complex regulatory system of respiratory enzymes, were increased in abundance in SD1 cells in vivo. FNR also activates sRNAs that degrade mRNAs coding for proteins involved in aerobic respiration [36]. This data supported the notion that major metabolic shifts in aerobic/anaerobic respiration were regulated at the post-transcriptional level. Periplasmic nitrate reductase, the Nap complex, was strongly increased in vivo upon comparing the abundances of the subunits NapA, NapB and NapC. In E. coli, Nap was shown to be induced under anaerobic conditions and also regulated by FNR and NarP [37].
Nap appears to act as an electron acceptor under low nitrate conditions in E. coli, suggesting a similar function in SD1. The nitrite reductase (NirB/NirD) was also increased in vivo. This complex has been associated with nitrite detoxification and appears to be metabolically linked to the activity of the periplasmic Nap protein.

Low abundance of electron donors of respiratory complexes was indicative of a switch to mixed acid fermentation in vivo. Indeed, proteomic evidence strongly supported the assumption that mixed acid fermentation and substrate level phosphorylation substituted for the low abundance of electron donors. Dramatic increases were noted for subunits of pyruvate formate lyase complexes. This included the activating enzyme PflA, formate acetyltransferases (PflB, TdcE), a putative formate acetyltransferase $\mathrm{YbiW}$, and the stress-induced alternate pyruvate formate lyase YfiD. Other mixed acid fermentation branches also appeared to be more active in vivo, such as the one initiated by PykA/PykF, which is coupled to acetate secretion via the phosphate acetyltransferase (Pta) and acetate kinase (AckA) activities. Interestingly, the fermentation/respiration switch protein FrsA was increased in abundance in vivo. In summary, this data provided comprehensive molecular evidence for the shift from aerobic/ microaerobic respiration to fermentation in SD1 cells in the host intestinal environment.

Fermentation pathways and associated stress responses have been characterized in E. coli [38]. The dramatic quantitative increase of YfiD is indicative of the fact that the glycyl radical protein is a key enzyme required to 
maintain the activity of PflA/PflB. YfiD has also been linked to low pH stress; the notion that this protein is essential for the survival of Shigella in the host gastrointestinal environment is intriguing, and makes YfiD a prospective drug target. The E. coli YfiD was also reported to be induced under acidic conditions in vitro [39]. The stress-induced alternate pyruvate formate-lyase YfiD appears to replace PflB upon oxidative inactivation during oxidative stress conditions in E. coli [40], thus supporting a critical metabolic role of the pyruvate-formate lyase PflA/YfiD in SD1 cells in vivo. Other mixed acid fermentation branches operating in vivo included reductive pathways for lactate and ethanol, each generating $\mathrm{NAD}+$ from NADH. In summary, survey of proteomic data supports strong activity increases in mixed acid fermentation, whereas the TCA cycle and aerobic processes were decreased correspondingly in SD1 cells localized in the anaerobic piglet intestine environment.

\section{Response of SD1 cells in vivo to acid stress and toxic conditions}

Acid resistance systems enable the survival of commensal and pathogenic E. coli strains in the acidic stomach and host intestinal environment, and neutralize intracellular acidic fermentation products $[41,42]$. Strong abundance changes of several SD1 enzymes contributing to $\mathrm{pH}$ homeostasis in this pathogen were identified in a recent study (15). This data lends further credence to the important function of two acid resistance (AR) systems, AdiA/ AdiC and $\mathrm{GadB} / \mathrm{GadC}$, to maintain the intracellular $\mathrm{pH}$ in SD1 cells during gastric passage and, possibly, as a result of increased generation of acidic fermentation products in the intestine. The orthologous AR systems were previously characterized in E. coli [42]. While increases of AdiA were strong in vivo, they also revealed variability comparing the piglet-derived samples (Additional File 3, Table S3). Of the transcription factors GadX, EvgA and $\mathrm{YdeO}$, all reported to influence expression of acid resistance genes [42,43], EvgA was increased in vivo suggesting a key regulatory role of EvgA during acidic stress in SD1. As also reported earlier (15), two periplasmic acid resistance chaperones (HdeA, HdeB) which protect periplasmic proteins from aggregation and denaturation at low $\mathrm{pH}$ were increased in SD1 cells in vivo. Hde proteins expose hydrophobic protein surfaces at low $\mathrm{pH}$ and initiate formation of aggregates with denatured periplasmic protein substrates $[44,45]$.

Host invasion by SD1 implicates the invasion and release from gut epithelial cells and cells of the innate and adaptive immune systems. SD1 cells are exposed to toxic molecules produced and secreted by cells of the immune system. We mined proteomic data for indicators of the molecular response to toxins. The most intriguing finding was the high abundance of nitric oxide
(NO) dioxygenase (HmpA) detected only under in vivo conditions. NO is known to be produced in large quantities in macrophages and is toxic to intracellular bacteria. In $M$. tuberculosis, the nitric oxide dioxygenase $\mathrm{HbN}$ was shown to be important for nitrite detoxification [46]. A hydroxylamine reductase, $\mathrm{YbjW}$, also scavenges toxic by-products of nitrogen metabolism and was detected only in vivo in SD1 cells. We speculate that the expression of these SD1 enzymes reflected memory of a previous intracellular life stage. Both enzymes are interesting targets for inhibitory drug design. To cope with oxidative stress, SD1 cells displayed increased abundances of superoxide dismutases (SOD) whose expression has been linked to oxygen availability in E. coli in vivo [47]. The previously mentioned regulator FNR and FNR-dependent small RNAs appear to be implicated in oxygen-dependent SOD abundance changes [48]. SodA and SodC were decreased in vivo, while iron-dependent SodB was clearly increased in vivo. An increase was noticed as well for the alkyl hydroperoxide reductase subunits AhpF/AhpC in vivo. The AhpC/AhpF subunits have also been implicated in the $S$. typhimurium defense in the macrophage environment [49]. The immune system in higher organisms including mice and humans generates reactive oxygen species, such as superoxide and peroxide ions to destroy invading microbes [50,51]. The increased abundance of oxidative stress proteins in vivo therefore implied a link to bacterial survival through evasion of the host immune response targeted against intestinal pathogens. Among the most dramatic abundance changes were those of three cold shock proteins (CspA, $\mathrm{CspC}$ and CspE). While the exact functions of these low $M_{r}$ proteins are not known, a recent study suggested that $c s p$ gene mutants reduced Listeria monocytogenes invasiveness [52], raising the question of their roles in epithelial or macrophage cell invasion by SD1. The dramatic abundance change of CspA (in vivo vs. in vitro) makes this protein a particularly interesting target for further characterization. Heat shock proteins (DnaK, IbpA, HtpG, GrpE) and chaperones (HslU/HslV, ClpB/ $\mathrm{ClpX)}$ were also increased, indicative of further intracellular stress responses by the SD1 cells in vivo. In summary, we gained insight into protein expression changes likely required for the survival of $S$. dysenteriae during oxidative and acid stress in the host intestine.

\section{SD1 outer membrane and cell surface proteome}

A large number of known or predicted $\beta$-barrel OM proteins were altered in abundance comparing in vivo and in vitro samples (OmpA, OmpC, IcsP/OmpT, OmpW/YciD, YaeT, Tsx, Lpp). Many of those proteins are either known or assumed to be exposed at the cell surface. A large number of lipoproteins sorted into the 
OM were also decreased in abundance under in vivo conditions (YoaF, LolB, SlyB, YcfM, NlpB, YfgL, NlpD). Proteins comprising the outer membrane YaeT protein assembly complex were decreased in abundance (YaeT, $\mathrm{NlpB}, \mathrm{YfgL}$ ) suggesting that the rate of biosynthesis and incorporation of the OM proteins was substantially decreased in vivo. Some of the chaperones presumably involved in OM protein transit and folding (YraP, HlpA, YtfJ), all part of RpoE regulon, were also decreased in abundance in vivo supporting the notion of reduced $\mathrm{OM}$ proteome turnover and a stress environment very different from that of stationary phase cells in vitro. Furthermore, components of the OM lipid asymmetry complex (YrbD, YrbF) and its regulator YrbA were decreased in abundance in vivo. This complex is a phospholipid transporter responsible for the balance of phospholipids and lipopolysaccharides in the outer leaflet of the OM. The outer membrane protein Imp directing the assembly of lipopolysaccharides was also decreased, while LolD, involved in translocation of OM lipoproteins, and the lipoprotein Nlpl (YhbM) were detected only in vitro. These changes, structurally or functionally associated with the OM, suggest a remodeling of the OM-associated cell surface, comprised of lipids, lipopolysaccharides and surface proteins, and a decreased turnover of proteins in the OM. Interestingly, the transcription factor BolA which regulates the transcription of genes involved in the morphology of the cell to confer cell protection was substantially decreased in vivo. Two other proteins likely involved in cell morphology and peptidoglycan turnover were also decreased in abundance under in vivo conditions, the rod-shape determining membrane protein YfgA and the LysM domain protein YgaU. It remains to be demonstrated whether these changes represent a coordinated physiological response of SD1 cells to the hostile environment in the host gut, possibly promoting evasion from the immune system and lowering OM porosity for protection from any extracellular toxic substances released by the host.

\section{S. dysenteriae type III secretion system and other virulence factors}

The virulence plasmid encodes the $30 \mathrm{~kb}$ spa-mxi type III secretion system (TTSS) and invasion plasmid antigens (Ipa proteins) required for invasion of host cells [53]. The TTSS is comprised of a membrane-spanning protein complex which includes $c a .50$ proteins, including Mxi and Spa proteins involved in assembly and regulation of the TTSS, chaperones (IpgA, IpgC, IpgE and Spa15), transcription activators (VirF, VirB and MxiE), translocators (IpaB, IpaC and IpaD) and ca. 25 effectors $[8,54]$. Invasion is followed by entry of Shigella into colonic epithelium cells via the basolateral membrane.
Further bacterial invasion and lateral spreading of the bacteria within the colonic epithelium is mediated by host cell actin polymerization. The surface protein IcsA encoded by the virulence plasmid is responsible for actin-based motility required for intra- and inter-cellular spread of the bacteria [55]. Shigella manipulates the host innate and adaptive immune system via the Osp family of proteins [56].

In the present study, we identified many components of the TTSS, including $15 \mathrm{Mxi}$-Spa proteins and 16 effectors and their chaperones (Additional File 1, Table S1). The TTSS has been reported as being assembled with a few effectors and chaperones when cultured in vitro, and activated only after contact of bacteria with host cells [8]. Here, many TTSS proteins were identified in both the in vitro and in vivo datasets, including membrane associated Mxi and Spa proteins, Ipa effectors and Spa chaperones. Spa15 is a chaperone for the Osp family of effectors (OspC1, OspC2, OspC3) and also for the IpaA and IpgB2 effectors; while IpgC is a chaperone for IpaB and IpaC [8]. Activation of TTSS results in the induction of the transcription of genes encoding a second set of effectors under the control of MxiE and IpgC, including several spa genes. The OspC2 and OspC3 effectors and the IpgA and Spa32 proteins were detected only under in vivo conditions. Activation of the TTSS is followed by formation of the TTSS translocator pore which requires the IpaB, IpaC and IpaD effectors $[5,57]$. IpaB in particular induces apoptosis in host macrophages leading to inflammatory infection [58]. Several of the Ipa proteins (IpaA/B/C/D) were increased in vivo, while other TTSS effectors were either increased or detected solely in vivo (IpaJ, IpgD, IcsB, OspC2, OspC3, OspF, VirA). Additional plasmid-encoded proteins such as PhoN1 and PhoN2 were decreased in abundance in vivo. PhoN2 was reported to hydrolyze dNTPs and modulate the localization of IcsA at the bacterial cell surface [59]. OspC2, IpaB and VirB were identified as immunogenic when probed with a piglet antiserum in a $2 \mathrm{D}$ Western blot [15], suggesting that these proteins could form potential vaccine targets for the prevention of shigellosis. The Ipa proteins are known to be transiently associated with the cell surface and therefore are likely to contribute to the altered SD1 cell surface in the host gut environment. We assume that other proteins likely secreted via the TTSS (OspC2, OspC3) are at least transiently cell surface associated. Abundance changes of the TTSS virulence factors correlated well with the altered changes in the OM/cell surface proteins in vivo. We are tempted to speculate that the previously mentioned OM remodeling efforts benefit the adaptation of SD1 to the host cell invasion process via enhanced abundance of TTSS effectors in the cell envelope. However, our data do not support uniformly 
increased abundances of all detected TTSS proteins in the SD1 cell envelope in vivo.

The virulence of Shigella species is of the order S. dysenteriae $>$ S. flexneri $>$ S. sonnei $>$ S. boydii. SD1 infection has a limited diarrheal phase with a sudden onset of acute dysentery, which could be explained by the expression of the potent virulence factor Shiga toxin (Stx) [14]. Shiga toxin subunit A (StxA) was detected only in vitro, while Shiga toxin subunit B (StxB) was detected both in vitro and in vivo, with StxB increased in abundance in vitro. As Stx is a secretory protein [14], the abundance levels of this protein are not readily obvious from proteomic profiling of cell lysates. It is of interest to examine whether the Shigella T2SS secretes other virulence factors in addition to the Shiga toxin. T2SS subunits were of very low abundance in SD1 cells according to this survey.

Other proteins involved in Shigella pathogenicity are the $\mathrm{O}$-antigens which are highly diverse with at least 46 observed serotypes [2]. The variability of the $\mathrm{O}$-antigens has been brought into context with evasion of the host immune system [60]. The small SD1 plasmid-encoded galactosyltransferase $\mathrm{RfpB}$ involved in the O-antigen biosynthesis was detected only in vivo, while other enzymes such as RfaD were increased in vivo. Enzymes potentially known to contribute monosaccharides (galactose and rhamnose) to the biosynthesis of the $\mathrm{O}$-antigen sugars were also increased in vivo, including LacZ, GalE/K/M/T, $\mathrm{RfbC}$, MelA, ManA and KdsB. Further studies are necessary to determine whether increased carbohydrate metabolism is functionally coupled to altered biosynthesis of $\mathrm{O}$-antigen sugars under in vivo conditions.

\section{Conclusions}

The comparative global proteomic survey of $S$. dysenteriae strain Sd1617 grown in vitro vs. S. dysenteriae cells isolated from an infected host animal model (in vivo) revealed abundance increases of several TTSS proteins and effectors under in vivo conditions. Virulence proteins such as OspC2 and IpaB, increased in abundance in vivo, were previously determined to be immunogenic, indicating their potential as vaccine candidates to combat shigellosis. Proteins important for the structural integrity of the bacterial cell wall and outer membrane such as OM proteins, lipoproteins, and chaperones for the cell envelope structures were decreased in vivo, indicating morphological changes in the bacterial cell wall. This hypothesis needs to be explored further in the context of infection, pathogenicity and protection from host factors. Proteins involved in response to anaerobic and nutrient deficient conditions, oxidative stress and acid stress were increased in vivo, reflecting the importance of the biochemical processes permitting the survival of the pathogen in the complex host gut environment. Further characterization of proteins increased in abundance in vivo will contribute to the understanding of host-pathogen interactions and facilitate the design of new vaccine candidates. It remains to be determined how the absence of microflora in the intestinal milieu might impact these observations.

\section{Additional material}

\begin{abstract}
Additional file 1: Table S1. Protein abundance estimates from APEX quantitation. APEX abundance values of 1761 S. dysenteriae serotype 1 (SD1) in vitro and in vivo proteins quantitated at a $<5 \%$ false discovery rate using the APEX Quantitative Proteomics Tool are listed along with their pi, ni, and Oi values. The corresponding gene names, locus tags, physicochemical properties and subcellular localizations are also listed in the table.
\end{abstract}

Additional file 2: Table S2. SD1 differential protein expression statistical analysis using Z-test and SAM. SD1 proteins listed in blue are upregulated under in vitro conditions. For the two tailed Z-test, SD1 proteins differentially expressed at 99\% confidence are listed; for the two class SAM test, proteins differentially expressed at $<10 \%$ FDR are listed.

Additional file 3: Table S3. APEX abundance values of SD1 in vitro and in vivo biological replicates. Three biological replicates from both in vitro and in vivo SD1 groups were analyzed as three to five technical replicates to expand the scope of the analysis; their APEX abundance values are listed.

\section{Abbreviations}

APEX: absolute protein expression; CM: cytoplasmic membrane; LPS: lipopolysaccharide; MS: mass spectrometry; OM: outer membrane; T3SS: type III secretion system; TCA: tricarboxylic acid; TMD: transmembrane domain.

\section{Acknowledgements}

We thank Dr. M. M. Venkatesan from the Walter Reed Army Institute of Research at Maryland, USA for kindly providing the Shigella dysenteriae serotype 1 Sd1617 strain. At Tufts, we thank D. Girouard for performing the animal C-sections. This part of the work was supported by the National Institute of Allergy and Infectious Diseases (NIAID), National Institutes of Health $(\mathrm{NIH})$ under contract number N01-Al-30050. At the JCVI, we thank T. Dracheva for helpful suggestions regarding bioinformatic tools for proteomic analysis, and S. Huang for submitting the SD1 proteomic datasets to the NCBI peptide data resource, Peptidome (Study PSE140 and Study PSE146). This part of the work was supported by the NIAID, NIH, under contract number N01-Al15447.

\section{Author details}

${ }^{1}$ Pathogen Functional Genomics Resource Center, J. Craig Venter Institute, 9704 Medical Center Drive, Rockville, MD 20850, USA. Division of Infectious Diseases, Cummings School of Veterinary Medicine, Tufts University, North Grafton, MA 01536, USA

\section{Authors' contributions}

SK - project conception and implementation, sample prep, generation of 2D-LC-MS/MS datasets and quantitation using the APEX Quantitative Proteomics Tool, bioinformatic, statistical and biological analyses of 2D-LCMS/MS-APEX datasets, primary manuscript author, QZ - provided bacterial samples, manuscript author, JCB - software engineering and development of the APEX Quantitative Proteomics Tool, statistical and pathway analysis of APEX datasets, manuscript review, AD - project oversight, provided bacterial samples, manuscript review, ST - project oversight, provided bacterial samples, manuscript review, RP - project conception and implementation, participation in data interpretation and writing of the manuscript. All authors read and approved the final manuscript.

\section{Competing interests}

The authors declare that they have no competing interests. 
Received: 24 July 2010 Accepted: 24 June 2011 Published: 24 June 2011

\section{References}

1. Niyogi SK: Shigellosis. J Microbiol 2005, 43(2):133-143.

2. Levine MM, Kotloff KL, Barry EM, Pasetti MF, Sztein MB: Clinical trials of Shigella vaccines: two steps forward and one step back on a long, hard road. Nat Rev Microbiol 2007, 5(7):540-553.

3. Shapiro RL, Kumar L, Phillips-Howard P, Wells JG, Adcock P, Brooks J, Ackers ML, Ochieng JB, Mintz E, Wahlquist S, Waiyaki P, Slutsker L: Antimicrobial-resistant bacterial diarrhea in rural western Kenya. J Infect Dis 2001, 183(11):1701-1704.

4. Herold S, Karch H, Schmidt H: Shiga toxin-encoding bacteriophagesgenomes in motion. Int J Med Microbiol 2004, 294(2-3):115-121.

5. Parsot C: Shigella spp. and enteroinvasive Escherichia coli pathogenicity factors. FEMS Microbiol Lett 2005, 252(1):11-18

6. Ogawa M, Handa Y, Ashida H, Suzuki M, Sasakawa C: The versatility of Shigella effectors. Nat Rev Microbiol 2008, 6(1):11-16.

7. Schroeder GN, Hilbi H: Molecular pathogenesis of Shigella spp.: controlling host cell signaling, invasion, and death by type III secretion. Clin Microbiol Rev 2008, 21(1):134-156.

8. Parsot C: Shigella type III secretion effectors: how, where, when, for what purposes? Curr Opin Microbiol 2009, 12(1):110-116.

9. Buchrieser C, Glaser P, Rusniok C, Nedjari H, D'Hauteville H, Kunst F, Sansonetti P, Parsot C: The virulence plasmid pWR100 and the repertoire of proteins secreted by the type III secretion apparatus of Shigella flexneri. Mol Microbiol 2000, 38(4):760-771.

10. Yao Z, Valvano MA: Genetic analysis of the O-specific lipopolysaccharide biosynthesis region (rfb) of Escherichia coli K-12 W3110: identification of genes that confer group 6 specificity to Shigella flexneri serotypes $Y$ and 4a. J Bacteriol 1994, 176(13):4133-4143.

11. Wei C, Yang J, Zhu J, Zhang X, Leng W, Wang J, Xue Y, Sun L, Li W, Jin Q: Comprehensive proteomic analysis of Shigella flexneri 2a membrane proteins. J Proteome Res 2006, 5(8):1860-1865.

12. Ying T, Wang H, Li M, Wang J, Wang J, Shi Z, Feng E, Liu X, Su G, Wei K, Zhang $X$, Huang $P$, Huang L: Immunoproteomics of outer membrane proteins and extracellular proteins of Shigella flexneri 2a 2457T. Proteomics 2005, 5(18):4777-4793.

13. Jennison AV, Raqib R, Verma NK: Immunoproteome analysis of soluble and membrane proteins of Shigella flexneri 2457T. World J Gastroenterol 2006, 12(41):6683-6688

14. Yang $F$, Yang J, Zhang $X$, Chen $L$, Jiang $Y$, Yan $Y$, Tang $X$, Wang J, Xiong $Z$, Dong J, Xue Y, Zhu Y, Xu X, Sun L, Chen S, Nie H, Peng J, Xu J, Wang Y, Yuan Z, Wen Y, Yao Z, Shen Y, Qiang B, Hou Y, Yu J, Jin Q: Genome dynamics and diversity of Shigella species, the etiologic agents of bacillary dysentery. Nucleic Acids Res 2005, 33(19):6445-6458.

15. Pieper R, Zhang Q, Parmar PP, Huang ST, Clark DJ, Alami H, DonohueRolfe A, Fleischmann RD, Peterson SN, Tzipori S: The Shigella dysenteriae serotype 1 proteome, profiled in the host intestinal environment, reveals major metabolic modifications and increased expression of invasive proteins. Proteomics 2009, 9(22):5029-5045.

16. Lu P, Vogel C, Wang R, Yao X, Marcotte EM: Absolute protein expression profiling estimates the relative contributions of transcriptional and translational regulation. Nat Biotechnol 2007, 25(1):117-124.

17. Kuntumalla S, Braisted JC, Huang ST, Parmar PP, Clark DJ, Alami H, Zhang Q, Donohue-Rolfe A, Tzipori S, Fleischmann RD, Peterson SN, Pieper R: Comparison of two label-free global quantitation methods, APEX and 2D gel electrophoresis, applied to the Shigella dysenteriae proteome. Proteome Sci 2009, 7:22.

18. Ross PL, Huang YN, Marchese JN, Williamson B, Parker K, Hattan S, Khainovski N, Pillai S, Dey S, Daniels S, Purkayastha S, Juhasz P, Martin S, Bartlet-Jones M, He F, Jacobson A, Pappin DJ: Multiplexed protein quantitation in Saccharomyces cerevisiae using amine-reactive isobaric tagging reagents. Mol Cell Proteomics 2004, 3(12):1154-1169.

19. Nesvizhskii Al, Keller A, Kolker E, Aebersold R: A statistical model for identifying proteins by tandem mass spectrometry. Anal Chem 2003, 75(17):4646-4658.

20. Keller A, Eng J, Zhang N, Li XJ, Aebersold R: A uniform proteomics MS/MS analysis platform utilizing open XML file formats. Mol Syst Biol 2005, 1, 2005.0017.

21. Braisted JC, Kuntumalla S, Vogel C, Marcotte EM, Rodrigues AR, Wang R, Huang ST, Ferlanti ES, Saeed Al, Fleischmann RD, Peterson SN, Pieper R: The
APEX Quantitative Proteomics Tool: generating protein quantitation estimates from LC-MS/MS proteomics results. BMC Bioinformatics 2008 , 9:529.

22. Vogel C, Marcotte EM: Calculating absolute and relative protein abundance from mass spectrometry-based protein expression data. Nat Protoc 2008, 3(9):1444-1451

23. Mallick P, Schirle M, Chen SS, Flory MR, Lee H, Martin D, Ranish J, Raught B, Schmitt R, Werner T, Kuster B, Aebersold R: Computational prediction of proteotypic peptides for quantitative proteomics. Nat Biotechnol 2007, 25(1):125-131

24. Gardy JL, Laird MR, Chen F, Rey S, Walsh CJ, Ester M, Brinkman FS: PSORTb v.2.0: expanded prediction of bacterial protein subcellular localization and insights gained from comparative proteome analysis. Bioinformatics 2005, 21(5):617-623.

25. Bendtsen JD, Nielsen $H$, von Heijne G, Brunak S: Improved prediction of signal peptides: SignalP 3.0. J Mol Biol 2004, 340(4):783-795.

26. Bendtsen JD, Nielsen $H$, Widdick D, Palmer T, Brunak S: Prediction of twinarginine signal peptides. BMC Bioinformatics 2005, 6:167.

27. Krogh A, Larsson B, von Heijne G, Sonnhammer EL: Predicting transmembrane protein topology with a hidden Markov model: application to complete genomes. J Mol Biol 2001, 305(3):567-580.

28. Berven FS, Flikka K, Jensen HB, Eidhammer I: BOMP: a program to predict integral beta-barrel outer membrane proteins encoded within genomes of Gram-negative bacteria. Nucleic Acids Res 2004, , 32 Web Server: W394-399.

29. Juncker AS, Willenbrock $H$, Von Heijne G, Brunak S, Nielsen H, Krogh A: Prediction of lipoprotein signal peptides in Gram-negative bacteria. Protein Sci 2003, 12(8):1652-1662.

30. Saeed Al, Sharov V, White J, Li J, Liang W, Bhagabati N, Braisted J, Klapa M, Currier T, Thiagarajan M, Sturn A, Snuffin M, Rezantsev A, Popov D, Ryltsov A, Kostukovich E, Borisovsky I, Liu Z, Vinsavich A, Trush V, Quackenbush J: TM4: a free, open-source system for microarray data management and analysis. Biotechniques 2003, 34(2):374-378.

31. Saeed Al, Bhagabati NK, Braisted JC, Liang W, Sharov V, Howe EA, Li J, Thiagarajan M, White JA, Quackenbush J: TM4 microarray software suite. Methods Enzymol 2006, 411:134-193.

32. Kanehisa M, Goto S: KEGG: kyoto encyclopedia of genes and genomes. Nucleic Acids Res 2000, 28(1):27-30.

33. Zhang Q, Donohue-Rolfe A, Krautz-Peterson G, Sevo M, Parry N, Abeijon C, Tzipori S: Gnotobiotic piglet infection model for evaluating the safe use of antibiotics against Escherichia coli 0157:H7 infection. J Infect Dis 2009, 199(4):486-493.

34. Jeong Kl, Zhang Q, Nunnari J, Tzipori S: A piglet model of acute gastroenteritis induced by Shigella dysenteriae Type 1. J Infect Dis 2010, 201(6):903-911.

35. Ying TY, Wang JJ, Wang HL, Feng EL, Wei KH, Huang LY, Huang PT, Huang CF: Immunoproteomics of membrane proteins of Shigella flexneri 2a 2457T. World I Gastroenterol 2005, 11(43):6880-6883.

36. Durand S, Storz G: Reprogramming of anaerobic metabolism by the FnrS small RNA. Mol Microbiol 2010, 75:1215.

37. McNicholas PM, Gunsalus RP: The molybdate-responsive Escherichia coli ModE transcriptional regulator coordinates periplasmic nitrate reductase (napFDAGHBC) operon expression with nitrate and molybdate availability. J Bacteriol 2002, 184(12):3253-3259.

38. Kirkpatrick C, Maurer LM, Oyelakin NE, Yoncheva YN, Maurer R, Slonczewski JL: Acetate and formate stress: opposite responses in the proteome of Escherichia coli. J Bacteriol 2001, 183(21):6466-6477.

39. Wyborn NR, Messenger SL, Henderson RA, Sawers G, Roberts RE, Attwood MM, Green J: Expression of the Escherichia coli yfiD gene responds to intracellular $\mathrm{pH}$ and reduces the accumulation of acidic metabolic end products. Microbiology 2002, 148(Pt 4):1015-1026.

40. Wagner AF, Schultz S, Bomke J, Pils T, Lehmann WD, Knappe J: YfiD of Escherichia coli and Y06l of bacteriophage T4 as autonomous glycyl radical cofactors reconstituting the catalytic center of oxygen-fragmented pyruvate formate-lyase. Biochem Biophys Res Commun 2001, 285(2):456-462.

41. Bearson S, Bearson B, Foster JW: Acid stress responses in enterobacteria. FEMS Microbiol Lett 1997, 147(2):173-180

42. Foster JW: Escherichia coli acid resistance: tales of an amateur acidophile. Nat Rev Microbiol 2004, 2(11):898-907.

43. Masuda N, Church GM: Regulatory network of acid resistance genes in Escherichia coli. Mol Microbiol 2003, 48(3):699-712. 
44. Kern R, Malki A, Abdallah J, Tagourti J, Richarme G: Escherichia coli HdeB is an acid stress chaperone. J Bacteriol 2007, 189(2):603-610.

45. Malki A, Le HT, Milles S, Kern R, Caldas T, Abdallah J, Richarme G: Solubilization of protein aggregates by the acid stress chaperones $\mathrm{HdeA}$ and HdeB. J Biol Chem 2008, 283(20):13679-13687.

46. Pathania R, Navani NK, Gardner AM, Gardner PR, Dikshit KL: Nitric oxide scavenging and detoxification by the Mycobacterium tuberculosis haemoglobin, $\mathrm{HbN}$ in Escherichia coli. Mol Microbiol 2002, 45(5):1303-1314

47. Hopkin KA, Papazian MA, Steinman HM: Functional differences between manganese and iron superoxide dismutases in Escherichia coli K-12. J Biol Chem 1992, 267(34):24253-24258

48. Boysen A, Moller-Jensen J, Kallipolitis B, Valentin-Hansen P, Overgaard M: Translational regulation of gene expression by an anaerobically induced small non-coding RNA in Escherichia coli. J Biol Chem 2010, 285(14):10690-10702

49. Hebrard M, Viala JP, Meresse S, Barras F, Aussel L: Redundant hydrogen peroxide scavengers contribute to Salmonella virulence and oxidative stress resistance. J Bacterio/ 2009, 191(14):4605-4614.

50. Yue WF, Liu JM, Sun JT, Li GL, Li XH, Wu XF, Sun HX, Zhou JY, Miao YG: Immunity promotion and proteomic identification in mice upon exposure to manganese superoxide dismutase expressed in silkworm larvae. J Proteome Res 2007, 6(5):1875-1881.

51. Bergin D, Reeves EP, Renwick J, Wientjes FB, Kavanagh K: Superoxide production in Galleria mellonella hemocytes: identification of proteins homologous to the NADPH oxidase complex of human neutrophils. Infect Immun 2005, 73(7):4161-4170.

52. Loepfe C, Raimann E, Stephan R, Tasara T: Reduced Host Cell Invasiveness and Oxidative Stress Tolerance in Double and Triple csp Gene Family Deletion Mutants of Listeria monocytogenes. Foodborne Pathog Dis 2010

53. Tamano K, Aizawa S, Katayama E, Nonaka T, Imajoh-Ohmi S, Kuwae A, Nagai S, Sasakawa C: Supramolecular structure of the Shigella type III secretion machinery: the needle part is changeable in length and essential for delivery of effectors. EMBO J 2000, 19(15):3876-3887.

54. Hueck CJ: Type III protein secretion systems in bacterial pathogens of animals and plants. Microbiol Mol Biol Rev 1998, 62(2):379-433.

55. Bernardini ML, Mounier J, d'Hauteville H, Coquis-Rondon M, Sansonetti PJ: Identification of icsA, a plasmid locus of Shigella flexneri that governs bacterial intra- and intercellular spread through interaction with F-actin. Proc Natl Acad Sci USA 1989, 86(10):3867-3871.

56. Zurawski DV, Mumy KL, Faherty CS, McCormick BA, Maurelli AT: Shigella flexneri type III secretion system effectors OspB and OspF target the nucleus to downregulate the host inflammatory response via interactions with retinoblastoma protein. Mol Microbio/ 2009, 71(2):350-368.

57. Picking WL, Nishioka $H$, Hearn PD, Baxter MA, Harrington AT, Blocker A, Picking WD: IpaD of Shigella flexneri is independently required for regulation of Ipa protein secretion and efficient insertion of IpaB and IpaC into host membranes. Infect Immun 2005, 73(3):1432-1440.

58. Sansonetti PJ: Microbes and microbial toxins: paradigms for microbialmucosal interactions III. Shigellosis: from symptoms to molecular pathogenesis. Am J Physiol Gastrointest Liver Physiol 2001, 280(3):G319-323.

59. Santapaola D, Del Chierico F, Petrucca A, Uzzau S, Casalino M, Colonna B, Sessa R, Berlutti F, Nicoletti M: Apyrase, the product of the virulence plasmid-encoded phoN2 (apy) gene of Shigella flexneri, is necessary for proper unipolar IcsA localization and for efficient intercellular spread. J Bacteriol 2006, 188(4):1620-1627.

60. Liu B, Knirel YA, Feng L, Perepelov AV, Senchenkova SN, Wang Q, Reeves PR, Wang L: Structure and genetics of Shigella $\mathrm{O}$ antigens. FEMS Microbiol Rev 2008, 32(4):627-653

doi:10.1186/1471-2180-11-147

Cite this article as: Kuntumalla et al.: In vivo versus in vitro protein abundance analysis of Shigella dysenteriae type 1 reveals changes in the expression of proteins involved in virulence, stress and energy metabolism. BMC Microbiology 2011 11:147

\section{Submit your next manuscript to BioMed Central and take full advantage of:}

- Convenient online submission

- Thorough peer review

- No space constraints or color figure charges

- Immediate publication on acceptance

- Inclusion in PubMed, CAS, Scopus and Google Scholar

- Research which is freely available for redistribution

Submit your manuscript at www.biomedcentral.com/submit
Biomed Central 